\title{
BMJ Open Protocol for a feasibility exploratory multicentre study of factors influencing trauma patients' outcomes of traffic crashes in Saudi Arabia
}

\author{
Rayan Alharbi (10 , ${ }^{1,2}$ Charne Miller, ${ }^{3}$ Virginia Lewis ${ }^{1}$
}

To cite: Alharbi R, Miller C, Lewis V. Protocol for a feasibility exploratory multicentre study of factors influencing trauma patients' outcomes of traffic crashes in Saudi Arabia. BMJ Open 2019;9:e032046. doi:10.1136/ bmjopen-2019-032046

- Prepublication history and additional material for this paper are available online. To view these files, please visit the journal online (http://dx.doi org/10.1136/bmjopen-2019032046).

Received 25 June 2019 Revised 05 September 2019 Accepted 17 September 2019

Check for updates

C Author(s) (or their employer(s)) 2019. Re-use permitted under CC BY-NC. No commercial re-use. See rights and permissions. Published by BMJ.

${ }^{1}$ Australian Institute for Primary Care and Ageing, School of Nursing \& Midwifery, La Trobe University, Bundoora, Victoria, Australia

${ }^{2}$ Department of Emergency Medical Service, Jazan University, Jazan, Saudi Arabia ${ }^{3}$ La Trobe Alfred Health Clinical School, La Trobe University, Prahran, Victoria, Australia

Correspondence to

Rayan Alharbi;

r.alharbi@latrobe.edu.au

\section{ABSTRACT}

Introduction Road traffic injury is a leading cause of death for people of all ages. The burden of road traffic injuries is well established in developed countries. However, there has been limited investigation of the incidence and burden of road traffic injury in low/middleincome countries. With a proportionally high number of road users, there is a need to explore the factors in prehospital and hospital care in Saudi Arabia (SA) that are associated with mortality for adult trauma patients following road traffic crashes (RTCs). This paper outlines the method for the planned research.

Methods and analysis A feasibility exploratory multicentre study will be conducted at three purposefully selected hospitals with different trauma care resources in differing geographic locations of SA. The study sample will include all adult trauma patients who are involved in RTCs in SA and have been admitted to a study site in a 3-month period from May to July 2019. Data regarding the characteristics of the crashes and prehospital health care factors will be extracted from hospital databases where it is available. Information will be collected from patients or carers and hospital records in the two sites that do not have a registry. Patient status at 30 days post-injury, particularly mortality, will be assessed through hospital records. The relative contribution of a range of factors to predicting mortality will be explored using logistic regression analysis.

Ethics and dissemination This study was approved by the Institutional Review Board Committee at King Saud Medical City (H-01-R-053), the General Department of Research and Studies at the Ministry of Health in SA (1440-1249939) and (1440-1398648), and the La Trobe University Human Research Ethics Committee (HEC19095). The results will be reported in a thesis and in peerreviewed journal articles and conference presentations.

\section{BACKGROUND}

Road traffic injury is a leading cause of death for people of all ages. The burden of road traffic injuries is well established in developed countries such as Australia, USA and Europe such as France, Germany, and Great Britain. ${ }^{1-3}$ However, there has been limited investigation of the incidence and burden in low/middle-income countries despite a

\section{Strengths and limitations of this study}

- This study will be conducted at three purposefully selected hospitals with different trauma resources in different geographic regions of Saudi Arabia (SA).

- This investigation will explore the factors in prehospital and hospital care that are associated with mortality for adult trauma patients following a road traffic crash (RTC).

- To our knowledge, this review will be the first population-based study using prehospital factors to predict RTC mortality in SA.

- This study may help to identify areas where efforts can be targeted to enhance the system of trauma care in SA.

- The feasibility of the study design will be considered and recommendations as to the conduct of large multicentre cohort studies in SA in future will be generated.

disproportionate degree of burden in these countries. For example, approximately $90 \%$ of the traumatic injury burden of total road traffic crashes (RTCs) occurs in low/ middle-income countries ${ }^{4}$ despite these countries having just $54 \%$ of the world's vehicles. ${ }^{5}$ A recent systematic review and meta-analysis involving 39 studies from 15 African countries estimated that there were 65.2 people injured per 100000 population in RTCs, with mortality of 16.6 deaths per 100000 population. ${ }^{6}$

The impact of RTCs is particularly evident in countries categorised as developing but with relatively high incomes such as Saudi Arabia (SA), as this often translates to more vehicles per capita. SA is one of the highest income countries in the Eastern Mediterranean according to $\mathrm{WHO}$ and income group. ${ }^{7}$ The burden of traumatic injury caused by RTCs is a major health problem and a leading cause of mortality and disability in SA. The country is ranked 23rd on the list of countries with the highest death rates in road crashes in 
the world.$^{8}$ It has been estimated that there are 19 deaths daily in the country and thousands disabled as a result of traffic-related injuries. ${ }^{9}$ In 2011, around 7153 people died and 39000 were injured in SA due to RTCs. ${ }^{10}$ Hence, efforts to reduce the prevalence and impact of RTCs in SA are warranted.

There have been few studies that have examined RTC deaths in different regions or hospitals with different trauma resources in SA to identify impacts of trauma care and potential for improvement. ${ }^{11} 12$ Moreover, no studies have been identified in the literature predicting RTC mortality based on prehospital processes, and the role of prehospital care on the outcomes of injured patients in SA is unknown. ${ }^{13}$ Investigations focusing on prehospital care would help describe usual care and identify factors associated with optimal outcomes. A study is proposed that will explore the factors in prehospital and hospital care that are associated with mortality for adult trauma patients following an RTC across different hospitals with different trauma resources in three regions of SA. In lieu of a national trauma registry, the capacity to capture standardised and comprehensive data from various institutions is unclear. As such, a feasibility exploratory multicentre study will inform the conduct of a larger national investigation. This paper describes the research method of such a feasibility exploratory multicentre study.

\section{METHODS}

\section{Study design}

A feasibility exploratory multicentre study will be conducted at three purposefully selected hospitals with different trauma care resources in different parts of SA including metropolitan and regional areas. Data collected by the King Saud Medical City (KSMC) trauma registry programme will be provided to the research team. The other two hospitals will collect comparable data during the same time period. As the patient's file at the two hospitals without a registry are not likely to include all of the required data fields, patients will be asked to give consent for the collection of additional data by the hospital staff. Data will be collected by local research partners (nurses/health professionals). The KSMC maintains hospital records (a trauma registry) that align with the study eligibility criteria and will be able to generate an electronic record of all patients who met the study criteria from the trauma registry. Variables related to the hospital, including level of resources available and length of stay, will be collected from the three sites.

The WHO promotes a definition of road traffic death that includes people who die up to 30 days after a crash. ${ }^{5}$ Presently, over 100 countries use this definition.

The use of 30-day in-hospital mortality as an outcome measure is common, ${ }^{14-17}$ as is exclusion of patients who were dead on arrival or died early (within $30 \mathrm{~min}$ to 1 hour of arrival) in the emergency room. ${ }^{15-20}$ The reliability of the indicator is supported by research that shows that the majority of trauma patients died within 30 days after injury $^{21}$ and the number of deaths post-discharge is very low. ${ }^{22}$ Further, death occurring after 30 days following trauma is more likely to be caused by other conditions. ${ }^{16}$

In this study patient status at 30-day post-crash, particularly mortality, will be assessed through hospital records. The relative contribution of a range of factors to predicting mortality will be explored. The feasibility of the study design to generate comprehensive data will be assessed.

\section{Patient and public involvement}

Patients are not directly involved in the design and conception of this study.

\section{Study sample}

Consecutive sampling is defined as a non-probability sampling technique where every patient who meets the criteria for inclusion is selected until the required sample size is achieved. This sampling approach is most appropriate for small exploratory quantitative studies. ${ }^{23}$ The study sample will include all adult (18 years old) trauma patients who are involved in an RTC in SA and who have been admitted to the three selected hospitals from 1 May 2019 to 31 July 2019. To ensure the representation of facilities with varying levels of trauma service, thus enhancing the generalisability of the study findings, the study will include three large to medium healthcare facilities from metropolitan and regional areas of SA. Specifically, hospitals included in the proposed study are KSMC in Riyadh (metropolitan area), Buraidah Central Hospital (metropolitan area) and Yanbu General Hospital in Yanbu (regional area).

With an intent to conduct a logistic regression analysis, a cases to independent variables (IV) ratio recommended by Tabachnick and Fidell ${ }^{24}$ was determined by the formula $\mathrm{n} \geq 50+8 \mathrm{~m}$ where $\mathrm{m}$ is the number of IVs. With the inclusion of up to 25 predictive variables a sample greater than 250 cases is required. In terms of the expected mortality, previous studies in SA have reported mortality rates of $8.5 \%,{ }^{11} 9.5 \%{ }^{25}$ and $13 \% .^{26} \mathrm{~A}$ sample of $\mathrm{n}=600$ will be sought which, applying a mortality rate of $8.5 \%$, is anticipated to result in sufficient numbers of deaths to enable the proposed analyses to be conducted $(n=51)$.

A recent study examining the relationship between transportation mode and in-hospital complications at Medical City (with a bed capacity of 1500) in Riyadh, SA achieved a sample of 493 injured trauma patients from January to June $2016 .{ }^{27}$ In our study, one of the three targeted hospitals is KSMC which has a bed capacity of 1400 , with a lower bed capacity at the two other study sites. Collectively, it is expected that 3 months of data collection involving the three different hospitals will achieve the target sample.

\section{Eligibility criteria}

For an individual to be eligible for this research study they must satisfy the following inclusion criteria:

1. Adult (aged 18 years or more). 
2. Involved in an RTC in SA.

3. Admitted to one of the three selected hospitals from 1 May 2019 to 31 July 2019.

\section{Exclusion criteria}

1. Patient was deemed dead on arrival at the hospital.

2. Patient age $<18$ years.

\section{Measures}

Data regarding the characteristics of the crashes and prehospital healthcare factors will be extracted from hospital databases where it is available. Information will be collected from patients or carers and hospital records in the two sites that do not have a registry. Variables related to the care provided in hospital, including level of resources available and patient's length of stay, will also be collected. Patient status at 30 days post-admission, particularly mortality, will be assessed through hospital records. The variables to be collected are described in table 1 . The data collection tool is a structured document with tick boxes for response categories.

The feasibility-based assessments of the study method to conduct future large multicentre cohort studies will include the rate of recruitment and reasons for participant ineligibility, time to screen patients and time required

Table 1 Shown complete study measurement

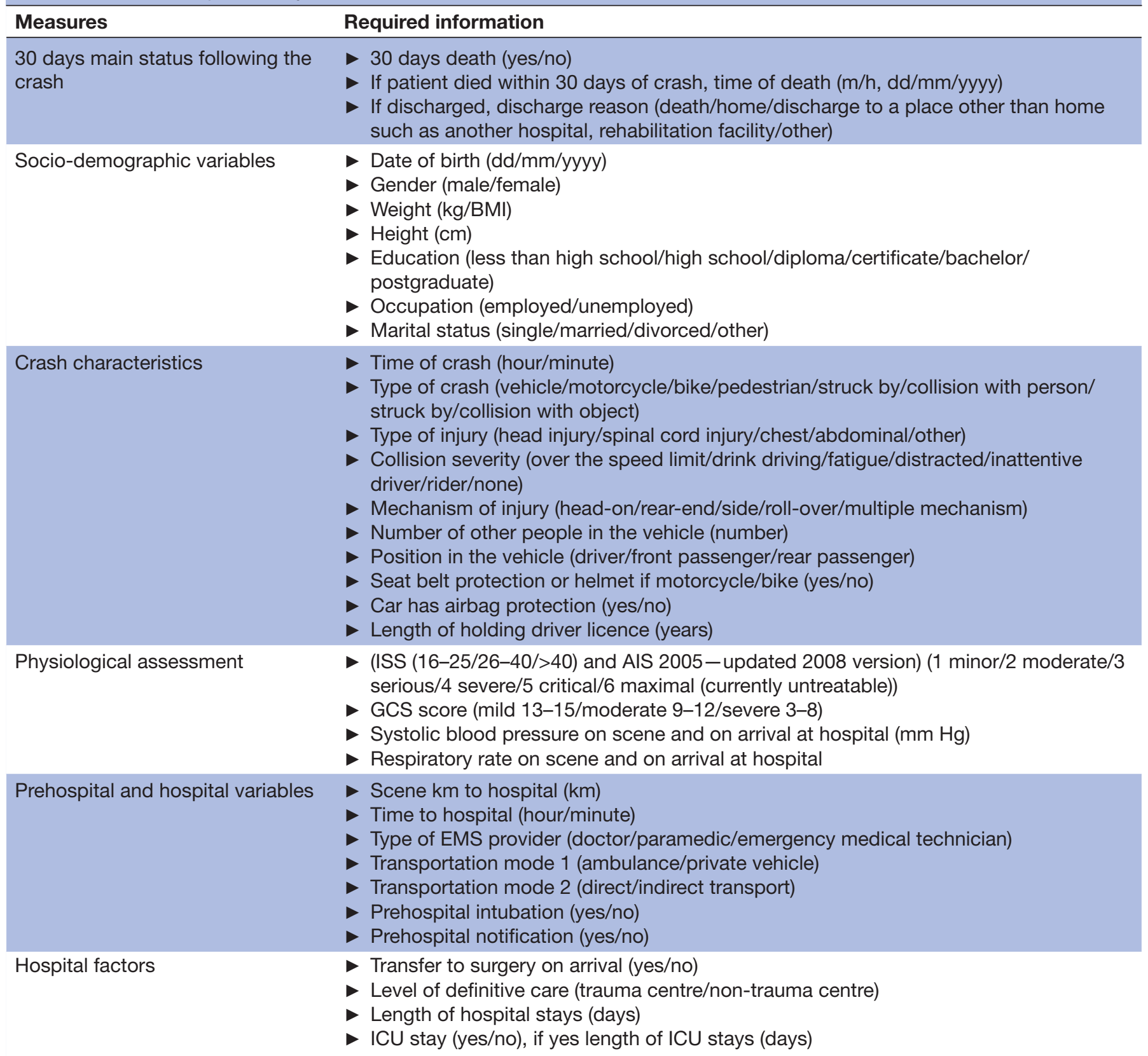

AIS, Abbreviated Injury Score; BMI, body mass index; EMS, emergency medical service; GCS, Glasgow Coma Scale; ICU, intensive care unit; ISS, Injury Severity Score. 
to collect data. Other feasibility-based assessments will include equipment and process requirements to implement the study, the existence of patient databases and minimum data fields, the number and type of sites that would need to be involved to achieve sample size targets and requirements for training staff to collect data (eg, including the Abbreviated Injury Score (AIS)). Moreover, examining the amount, type and pattern of missing data is a focus for the initial steps in this feasibility study, the results of which will support the conduct of subsequent multicentre research about road trauma in SA. We also hope to explore potential differences in systems and practice that may contribute to outcomes.

\section{Statistical analyses}

Logistic regression analysis modelling, which is a form of predictive modelling technique, is suitable for this study. 'Logistic regression predicts the probability of an event occurring and is expressed as an OR' (p. 232). ${ }^{23}$ Incidence rates, OR and 95\% CI for the dependent variable (DV) (the target) and IVs will be calculated. The study will use $\chi^{2}$ tests, independent t-tests or Mann-Whitney $\mathrm{U}$ tests for univariate analyses. The DV of 30 days status includes death, in hospital, or discharged. Mortality will be adjusted for different IVs including socio-demographic variables, crash characteristics, physiological assessment, and prehospital and hospital variables. Due to the large number of IVs, some variables will be controlled in the regression analysis including age and Injury Severity Score (ISS). In addition to logistic regression, Cox proportional hazard model will be used to examine the relationship between the survival time of injured patients and explanatory variables, pending the availability of both mortality data and days to death data. ${ }^{28}$

Furthermore, as a feasibility study, a comparison of data quality (ie, the quantity and nature of missing data) will be considered. Sub-group analyses can be conducted to assess site differences in the quality of data. All analyses will be performed using the latest version of MacOS Statistical Package for the Social Sciences; a $p$-value $<0.05$ will be considered significant for all statistical tests. Other information about the feasibility of the study design will be reported such as recruitment time, need for resources to support data collection and any other difficulties/ limitations that arose during the study process.

\section{Patient consent}

Patient consent is not required for the KSMC site as the study will be conducted utilising de-identified data from the KSMC Trauma Registry, which has prior consent to be used for research. However, we have designed a consent form in two languages (Arabic and English) for the Buraidah Central Hospital and Yanbu General Hospital to use when collecting data for this study. These two sites have agreed to provide de-identified patient data for the study. All participants who meet the study inclusion criteria will be asked to answer questions related to the crash by local research partners (nurses/health professionals) at these two hospitals. Interviews will take approximately 10 min of the participant's time. Other information will be obtained from their prehospital records (provided by ambulance) and hospital file, with their consent. For patients who are not able to consent because of their injuries, next of kin will be asked to provide consent on behalf of these participants. In cases where a patient dies prior to the health professional researcher approaching them or their next of kin for consent, the researcher will ask the next of kin at an appropriate time when it is least likely to cause distress. A protocol is in place to support people who may become distressed; this involves linking the person to support services available in the hospital. All patients have the right to withdraw at any point. See online supplementary appendix 1 for full participant information statement and consent form.

\section{DISSEMINATION}

The results of this study will be reported in a thesis and in peer-reviewed journal articles and conference presentations. A final report and copy of any publication arising from the study will also be provided to KSMC, the Ministry of Health in SA and La Trobe University, Australia. The participating hospitals and Ministry of Health are able to share the final report/publications with participants or through other distribution mechanisms with appropriate acknowledgement.

\section{LIMITATIONS}

First, data can only be collected for injured patients who are admitted to one of the three selected hospitals as SA has no national trauma registry. Therefore, the three hospitals with different trauma care resources in different parts of SA have been purposefully selected, not only to provide a range of incident experiences and to test the study method in anticipation of larger national studies but also to enable documentation of mortality at 30 days post-crash as per the WHO definition. ${ }^{5}$ Second, patient status at 30 days post RTC admission is the key outcome measure. It is possible for someone to die in a different location before 30 days if patients are transferred elsewhere with ongoing medical issues, or they may die at home post-discharge. Therefore, there might be missing data for those who die elsewhere within the 30 days after discharge as hospitals will not follow-up with those who discharge. However, information about the reasons for discharge (such as home, discharge to another hospital, rehabilitation facility, or other) will be collected, and we will consider whether the transition represents a 'step up' in intensity of healthcare or a 'step down'. It is reasonable to assume that hospitals will only discharge people to a lower level of care or home if they are healthy enough.

Third, the use of AIS coding has been shown to be reliable when used by health professionals with different levels of training, particularly for vehicular injury compared with non-vehicular injury. ${ }^{29}$ While there exists 
a limitation in relation to knowing the levels of AIS training among staff who are documenting patients' information, hospital records in SA are based on information collected by trained administrators and nurses or health professionals. The local research partners (at the two prospective study sites) who will collect the study data from patients have at minimum a qualification of bachelor's in nursing or another health discipline. Further, the first author has provided instructions and ongoing advice to the hospital staff who are collecting data. The trauma registry data collection staff are qualified in use of the AIS.

\section{DISCUSSION}

This is primarily a feasibility study to examine the capacity to generate comprehensive and compatible data from multiple sites (hospitals) in SA. The overall aim of the research programme is to identify the factors that predict 30 day mortality in adult trauma patients following RTCs in SA, with a particular focus on prehospital and hospital processes in different hospitals with different trauma resources. The complementary research questions include, but are not limited to: Is it possible to conduct a large multicentre cohort study in SA?; what is the amount, type and pattern of missing data across study sites? (depending on quality and comprehensiveness of data); is there a difference in mortality between the three hospitals (controlling for severity)?, for example, metro versus regional; size of hospital; presence of trauma protocol; and is there a difference in the survival time of injured patients among the three hospitals?

The findings of this research will help to explore the preventability of death following RTCs and suggest improvements in the system of care provided to these patients in SA. In SA, the long-term outcomes and the predictive factors influencing mortality following RTCs are unknown. Only one recent study has investigated that long-term disabilities follow injuries; however, the study focused only on traumatic head injury patients in SA. ${ }^{30}$ Two other studies have examined factors predicting in-hospital death among patients injured in traffic crashes, but these studies were based on the only level I trauma centre (National Guard Hospital) in SA. ${ }^{11} 12$ These studies have shown some factors associated with higher risk of in-hospital death such as patients being older than 65 years, higher ISS and the mechanism of injury (pedestrians compared with motor vehicle crashes). ${ }^{11} 12$ In this study, we aim to assess the feasibility of using a multicentre research design to explore a comprehensive range of factors that could be associated with RTC patient mortality in prehospital and hospitals with different resources. An understanding of these predictive factors will help to identify areas where future efforts can be targeted to enhance the system of trauma care, explore the preventability of death and improve outcomes of patients injured in an RTC in SA.
Contributors This study protocol was developed by all authors. All authors approved the final version of the manuscript. RA prepared the first draft of this protocol paper and revised comments from CM and VL. All authors approved the final version of the manuscript.

Funding The authors have not declared a specific grant for this research from any funding agency in the public, commercial or not-for-profit sectors.

Competing interests None declared.

Patient consent for publication Not required.

Ethics approval This study was approved by the Institutional Review Board Committee at King Saud Medical City, SA (H-01-R-053). The Buraydah Central Hospital has approval from the General Department of Research and Studies (GDRS) at the Ministry of Health in SA (1440-1249939). Yanbu General Hospital has approval from the SA's Ministry of Health GDRS (1440-1398648). Finally, this study was approved by the La Trobe University Human Research Ethics Committee (HEC19095).

Provenance and peer review Not commissioned; externally peer reviewed.

Open access This is an open access article distributed in accordance with the Creative Commons Attribution Non Commercial (CC BY-NC 4.0) license, which permits others to distribute, remix, adapt, build upon this work non-commercially, and license their derivative works on different terms, provided the original work is properly cited, appropriate credit is given, any changes made indicated, and the use is non-commercial. See: http://creativecommons.org/licenses/by-nc/4.0/.

ORCID iD

Rayan Alharbi http://orcid.org/0000-0002-0504-3240

\section{REFERENCES}

1 Beckmann J, Beckmann J. Social and economic consequences of road traffic injury in Europe. J Beckmann-Brussels: European Transport Safety Council, 2007: 49.

2 Naumann RB, Dellinger AM, Zaloshnja E, et al. Incidence and total lifetime costs of motor vehicle-related fatal and nonfatal injury by road user type, United States, 2005. Traffic Inj Prev 2010;11:353-60.

3 Trawén A, Maraste P, Persson U. International comparison of costs of a fatal casualty of road accidents in 1990 and 1999. Accid Anal Prev 2002;34:323-32.

4 Ameratunga S, Hijar M, Norton R. Road-Traffic injuries: confronting disparities to address a global-health problem. Lancet 2006;367:1533-40.

5 WHO. Global status report on road safety 2015. World Health Organization, 2015.

6 Adeloye D, Thompson JY, Akanbi MA, et al. The burden of road traffic crashes, injuries and deaths in Africa: a systematic review and meta-analysis. Bull World Health Organ 2016;94:510-21.

7 WHO. Global status report on road safety Switzerland: World Health organization website, 2009. Available: http://apps.who.int/iris/ bitstream/handle/10665/44122/9789241563840_eng.pdf?sequence $=$ 1 \&isAllowed=y\&ua $=1$

8 Alarabiya. Report: Saudi Arabia records 526000 road accidents annually Jeddah. Saudi Arabia: Al Arabia News English, 2015. http:// english.alarabiya.net/en/News/middle-east/2016/01/01/ReportSaudi-Arabia-records-526-000-road-accidents-annually.html

9 Al Turki YA. How can Saudi Arabia use the decade of action for road safety to catalyse road traffic injury prevention policy and interventions? Int J Inj Contr Saf Promot 2014;21:397-402.

10 Alajlan S. Traffic injuries annual report of Saudi Arabia (2009). In: Traffic GDo. Saudi Arabia: Department of Traffic, 2011.

11 Alghnam S, Palta M, Hamedani A, et al. Predicting in-hospital death among patients injured in traffic crashes in Saudi Arabia. Injury 2014;45:1693-9.

12 Alghnam S, Palta M, Hamedani A, et al. In-Hospital mortality among patients injured in motor vehicle crashes in a Saudi Arabian Hospital relative to large U.S. trauma centers. Inj Epidemiol 2014;1.

13 Mansuri FA, Al-Zalabani AH, Zalat MM, et al. Road safety and road traffic accidents in Saudi Arabia. A systematic review of existing evidence. Saudi Med J 2015;36:418-24.

14 de Jongh MAC, Meeuwis JD, van Baar ME, et al. Evaluation of trauma care by comparing mortality risks and admission policy in a Dutch trauma region. Injury 2008;39:1007-12.

15 Di Bartolomeo S, Marino M, Ventura C, et al. Evaluation of the survival benefit of Trauma-Centre care in the Italian setting. Injury 2014;45:299-303. 
16 Bouamra O, Wrotchford A, Hollis S, et al. A new approach to outcome prediction in trauma: a comparison with the TRISS model. J Trauma 2006;61:701-10.

17 Garwe T, Cowan LD, Neas B, et al. Survival benefit of transfer to tertiary trauma centers for major trauma patients initially presenting to nontertiary trauma centers. Acad Emerg Med 2010;17:1223-32.

18 Moore L, Stelfox HT, Evans D, et al. Trends in injury outcomes across Canadian trauma systems. JAMA Surg 2017;152:168-74.

19 Siman-Tov M, Radomislensky I, Peleg K, et al. Reduction in trauma mortality in Israel during the last decade (2000-2010): the impact of changes in the trauma system. Injury 2013;44:1448-52.

20 MacKenzie EJ, Rivara FP, Jurkovich GJ, et al. A national evaluation of the effect of trauma-center care on mortality. N Engl J Med 2006;354:366-78.

21 Skaga NO, Eken T, Jones JM, et al. Different definitions of patient outcome: consequences for performance analysis in trauma. Injury 2008;39:612-22.

22 McDermott FT, Cordner SM, Cooper DJ, et al. Management deficiencies and death preventability of road traffic fatalities before and after a new trauma care system in Victoria, Australia. J Trauma 2007;63:331-8.
23 Schneider Z, Whitehead D, LoBiondo-Wood G. Nursing and midwifery research: methods and appraisal for evidence-based practice. 5th edn. Australia: Elsevier, 2016.

24 Tabachnick B, Fidell L. Using multivariate statistics (new international ed. ed.). Harlow: Pearson, 2014.

25 Alghnam S, Alkelya M, Al-Bedah K, et al. Burden of traumatic injuries in Saudi Arabia: lessons from a major trauma registry in Riyadh, Saudi Arabia. Ann Saudi Med 2014;34:291-6.

26 Aldawood AS, Alsultan M, Haddad S, et al. Trauma profile at a tertiary intensive care unit in Saudi Arabia. Ann Saudi Med 2012;32:498-501.

27 Aljerian N, Alhaidar S, Alothman A, et al. Association between the mode of transport and in-hospital medical complications in trauma patients: findings from a level-I trauma center in Saudi Arabia. Ann Saudi Med 2018;38:8-14.

28 Cox DR. Regression models and life-tables. J Royal Stat Soc 1972;34:187-202.

29 MacKenzie EJ, Shapiro S, Eastham JN. The abbreviated injury scale and injury severity score. levels of inter- and intrarater reliability. Med Care 1985;23:823-35.

30 Alghnam S, AlSayyari A, Albabtain I, et al. Long-Term disabilities after traumatic head injury (THI): a retrospective analysis from a large level-I trauma center in Saudi Arabia. Inj Epidemiol 2017;4. 